\title{
Status of HEP after the LHC Run 1
}

\section{Paraskevas Sphicas*}

European Center for Particle Physics and University of Athens

E-mail: paraskevas.sphicasecern.ch

In the past 20 years, the Standard Model (SM) of elementary particles and their interactions has provided an unfailing and remarkably accurate description of all experiments with and without high-energy accelerators, establishing that we understand the physics of the very small up to energy scales of $100 \mathrm{GeV}$. The Large Hadron Collider of CERN, and its experiments, were conceived to probe the physics of the next frontier, that of the $\mathrm{TeV}$ energy scale. True to their charge, the experiments have delivered hundreds of significant and often beautiful measurements, along with the discovery of what looks like the first fundamental scalar particle. The triumph of the Standard Model is complete, especially since no new signal has emerged from the intense searches for "new physics" - yet. The field is now at a crossroads: the existence of a Higgs boson opens a new set of questions, while the evidence, both direct and indirect, that there physics beyond the SM does exist, is still strong and convincing. The talk will present a broad-brush picture of how Run 1 of the LHC has shaped the field of High Energy Physics; along with why expectations are still so very high.

Frontiers of Fundamental Physics 14 - FFP14,

15-18 July 2014

Aix Marseille University (AMU) Saint-Charles Campus, Marseille

\footnotetext{
*Speaker.
} 